\title{
A bisection-based approach for exact target localization in NLOS environments
}

\author{
Slavisa Tomic ${ }^{\mathrm{a}, \mathrm{b}, *}$, Marko Beko ${ }^{\mathrm{b}, \mathrm{c}}$ \\ a ISR/IST, LARSyS, Universidade de Lisboa, 1049-001 Lisbon, Portugal \\ ${ }^{\mathrm{b}}$ CICANT-CIC.DIGITAL, Universidade Lusófona de Humanidades e Tecnologias, Campo Grande 376, 1749 - 024 Lisboa, Portugal \\ ${ }^{\mathrm{c}}$ CTS/UNINOVA, Campus da FCT/UNL, Monte de Caparica, 2829-516 Caparica, Portugal
}

\section{A R T I C L E I N F O}

\section{Article history:}

Received 13 June 2017

Revised 15 August 2017

Accepted 18 September 2017

Available online 20 September 2017

\section{Keywords:}

Target localization

Range-based localization

Non-line-of-sight (NLOS)

Generalized trust region sub-problem

(GTRS)

Wireless sensor network (WSN)

\begin{abstract}
A B S T R A C T
This work addresses the range-based target localization problem in adverse non-line-of-sight (NLOS) environments. We start by deriving the maximum likelihood (ML) estimator from the measurement model, since it is asymptotically efficient. However, this estimator is highly non-convex and difficult to solve directly. Hence, we convert the localization problem into a generalized trust region sub-problem (GTRS) framework. Although still non-convex in general, the derived estimator is strictly decreasing over a readily obtained interval, and thus, can be solved exactly by a bisection procedure. In huge contrast to existing algorithms, which either require the knowledge about the magnitude of the NLOS bias or to a priori distinguish between line-of-sight (LOS) and NLOS links, the new one does not require such prerequisites. Also, the computational complexity of the proposed algorithm is linear in the number of reference nodes, unlike the majority of existing ones. Our simulation results show that the new algorithm possesses a steady NLOS bias mitigation capacity and that it represents an excellent alternative in the sense of the trade off between accuracy and complexity. To be more specific, it not only matches the performance of existing methods (majority of which significantly more computationally complex) but outperforms them in general. Moreover, the performance of the proposed algorithm is validated through real-indoor experimental data.
\end{abstract}

(c) 2017 Elsevier B.V. All rights reserved.

\section{Introduction}

Target localization in indoor environments is of particular practical interest [1], since the use of global positioning system is not possible in such environments, and alternative means for localization are appreciated. A common approach in adverse indoor environments, where most or all connections are non-line-of-sight (NLOS), is to make use of reference nodes (called anchors) and utilize the (noise-corrupted) range measurements between the target and anchors. Range measurements can be extracted from different characteristics of the radio signal, such as time (difference) of arrival, time of flight, or received signal strength to name a few [2-10].

Nevertheless, the impact of NLOS bias might be great in adverse indoor environments, and it might deteriorate the localization performance. Therefore, different techniques for NLOS bias mitigation were proposed recently $[1,12-19]$. On the one hand, some of them

\footnotetext{
* Corresponding author.

E-mail addresses: stomic@isr.ist.utl.pt, s.tomic@campus.fct.unl.pt (S. Tomic), beko.marko@ulusofona.pt, mbeko@uninova.pt (M. Beko).
}

are based on the NLOS link identification [16,17], after which those links are either discarded or used in a certain manner to improve the localization performance. On the other hand, others are robust estimators $[11,13,19]$, which are generally more resilient to NLOS bias. A robust estimator based on semidefinite programming (SDP) relaxation technique was introduced in [11] to cope with the NLOS bias. Even though this estimator performs well in harsh indoor environments, its computational complexity is greatly affected by a growth in the network size. Another SDP estimator was presented in [12]. This estimator tries to mitigate the NLOS bias by properly handling the NLOS links, but disregards a relationship established between optimization variables. Besides, this estimator is $\gamma$ dependent, where $\gamma$ is a penalization term that prevents the problem to be ill-posed. In [13], a robust second-order cone programming (SOCP) estimator was described. Its computational burden is relatively low and it gives a decently accurate solution, but it is not always feasible. A perturbed edge-based SDP (PESDP) estimator was proposed in [14]. This estimator was originally designed for cooperative localization, and relies on perturbation terms introduced in target/target links to enhance its performance. In [18], a two-step adaptive weighted least squares (WLS) estimator for 
target localization in mixed line-of-sight (LOS)/NLOS environments was proposed. Although this estimator is fast and its accuracy is relatively high, its downside is that the identification between the LOS and NLOS is assumed to be perfectly known a priori, which might not stand in practice. A robust estimator based on generalized trust region sub-problem (GTRS) framework was presented in [19]. This estimator has linear computational complexity in the number of reference points, but it assumes that perfect knowledge about the upper bound on the magnitude of the NLOS bias is available, which might not be the case in practice.

Most of the above described algorithms for range-based target localization in NLOS environments [11-14] employ sophisticated mathematical tools which raise severely their computational complexity, and thus, their execution time. In huge contrast to them, here, we propose a novel estimator whose solution is obtained $e x$ actly by nothing other than a bisection procedure. In sharp contrast to some existing algorithms [11-13,18,19], the proposed algorithm does not require to know the upper bound on the magnitude of the NLOS bias, nor assumes knowledge about the statistics of the measurement noise. Moreover, it does not require to distinguish between LOS and NLOS links, nor to know the statistics of NLOS bias. We take the NLOS bias as a nuisance parameter, and develop a novel estimator that mitigates its influence. By applying a squared-range (SR) and WLS criterion, we convert the originally non-convex problem into a GTRS framework, whose solution is readily obtained by a bisection procedure [20]. Unlike most of the above described algorithms, computational complexity of the new one is linear in the number of anchors, and its performance is highly competitive with the state-of-the-art algorithms.

\section{Problem formulation}

Denote by $\boldsymbol{x}, \boldsymbol{a}_{i} \in \mathbb{R}^{k}$ the unknown location of the target and the known location of the $i$-th anchor, $i=1, \ldots, N$ in a $k$-dimensional WSN ( $k=2$ or 3 ), respectively. Then, distance measurements between the target and the $i$-th anchor in NLOS environments [11-14,19], can be modeled as

$d_{i}=\left\|\boldsymbol{x}-\boldsymbol{a}_{i}\right\|+b_{i}+n_{i}$, for $i=1, \ldots, N$,

where $n_{i}$ is the measurement noise, assumed to follow a zeromean Gaussian distribution with variance $\sigma_{i}^{2}$, i.e., $n_{i} \sim \mathcal{N}\left(0, \sigma_{i}^{2}\right)$, and $b_{i}$ is the NLOS bias $\left(b_{i}=0\right.$ if $i \in \mathcal{L}_{l}$ and $b_{i}>0$ if $i \in \mathcal{L}_{n}$, with $\mathcal{L}_{l}$ and $\mathcal{L}_{n}$ representing the set of all LOS and NLOS links, respectively).

In the literature, the NLOS bias is generally assumed to be positive and greater than the measurement noise [11-14,19]. Furthermore, in some works, authors have assumed that it follows a certain distribution, such as Gaussian, uniform or exponential to name a few. Also, an assumption that the magnitude of the NLOS bias is upper bounded by a known constant, i.e., $b_{i} \leq b_{\max }$ for $i=1, \ldots, N$ was made in some existing work $[11,13,19]$. Here, we do not make any assumptions of that sort about the NLOS bias, which gives a desirable property of universal applicability to our estimator, under the assumption of any distribution.

Given the observation vector $\boldsymbol{d}=\left[d_{i}\right]^{T}\left(\boldsymbol{d} \in \mathcal{R}^{N \times 1}\right)$, the probability density function (PDF) is written as

$p(\boldsymbol{d} \mid \boldsymbol{\theta})=\prod_{i=1}^{N} \frac{1}{\sqrt{2 \pi \sigma_{i}^{2}}} \exp \left\{-\frac{\left(d_{i}-\left\|\boldsymbol{H}^{T} \boldsymbol{\theta}-\boldsymbol{a}_{i}\right\|-\boldsymbol{h}^{T} \boldsymbol{\theta}\right)^{2}}{2 \sigma_{i}^{2}}\right\}$,

where $\quad \boldsymbol{\theta}=\left[\boldsymbol{x}^{T}, b_{i}\right]^{T}, \quad \boldsymbol{\theta} \in \mathbb{R}^{(k+N) \times 1}, \quad \boldsymbol{H}=\left[\boldsymbol{I}_{k}, \mathbf{0}_{k \times N}\right]^{T} \quad$ and $\quad \boldsymbol{h}=$ $\left[\mathbf{0}_{1 \times k}, \boldsymbol{e}_{i}^{T}\right]^{T}$, with $\boldsymbol{I}_{M}$ and $\boldsymbol{0}_{M \times M}$ representing the $M \times M$ identity matrix and matrix of all zero entries respectively, and $\boldsymbol{e}_{i}$ is the $i$ th column of $\boldsymbol{I}_{N}$.

Maximizing the log of the likelihood function in (2) with respect to $\boldsymbol{\theta}$, yields the maximum likelihood (ML) estimator
[21] of $\boldsymbol{\theta}, \widehat{\boldsymbol{\theta}}$

$\widehat{\boldsymbol{\theta}}=\underset{\boldsymbol{\theta}=\left[\boldsymbol{x}^{T}, b_{i}\right]^{T}}{\arg \min } \sum_{i=1}^{N} \frac{\left(d_{i}-\left\|\boldsymbol{H}^{T} \boldsymbol{\theta}-\boldsymbol{a}_{i}\right\|-\boldsymbol{h}^{T} \boldsymbol{\theta}\right)^{2}}{\sigma_{i}^{2}}$.

The problem in (3) is highly non-convex and has no closedform solution. Also, in practice, it is very difficult to establish which links are LOS and which are NLOS (thus increasing the probability of false alarm and misdetection). Therefore, some approximations are needed in order to solve (3). In Section 3, we present a detailed derivation of a novel estimator that is a tight approximation of the ML one, but whose exact solution is readily acquired by means of a bisection procedure.

\section{The proposed estimator}

Note that the problem defined by the equation given in (3) is under-determined, since the number of unknowns is greater than the number of observations. Hence, instead of tackling (3) directly, we start by approximating (1) by

$d_{i}=\left\|\boldsymbol{x}-\boldsymbol{a}_{i}\right\|+b+n_{i}$, for $i=1, \ldots, N$,

where we approximated the N NLOS biases by only one, $b$. This approximation was done in order to make the originally underdetermined problem a determined one (for $N \geq k+1$ in a $k$ dimensional space). Although in this way we can only partially mitigate the NLOS bias, this approach allows us to keep the parameter $b$ (called a balancing parameter) as an optimization variable which we will estimate alongside with the target location. In contrast to the existing robust approaches, e.g. the ones in $[11,13,19]$, where all links were considered as NLOS ones and a certain term was subtracted from every link (which introduces an error for all LOS links and possibly for some NLOS ones with small bias terms), here, we take a different approach. We estimate the mean bias from all links, avoiding the introduction of the error typical for the robust approaches. Therefore, the proposed approach still leaves us with somewhat control over the two extreme cases (all LOS/NLOS links), since it is possible to get a good estimation of the NLOS bias in these two cases, while the robust approach is expected to under-perform in the case of all LOS links (because it treats all links as NLOS) and perform well when all links are NLOS. In the case of half LOS and half NLOS links, the influence of the NLOS bias will be only partially mitigated with both approaches.

Then, by rearranging and squaring (4), we get

$\left(d_{i}-b\right)^{2}=\left\|\boldsymbol{x}-\boldsymbol{a}_{i}\right\|^{2}+2 n_{i}\left\|\boldsymbol{x}-\boldsymbol{a}_{i}\right\|+n_{i}^{2}$.

From (5), we can write

$\frac{\left(d_{i}-b\right)^{2}-\left\|\boldsymbol{x}-\boldsymbol{a}_{i}\right\|^{2}}{2\left\|\boldsymbol{x}-\boldsymbol{a}_{i}\right\|}=n_{i}+\frac{n_{i}^{2}}{2\left\|\boldsymbol{x}-\boldsymbol{a}_{i}\right\|}$.

Note that by squaring (4) the measurement noise is not Gaussian anymore. Nevertheless, for sufficiently low noise power, we can disregard the second-order noise term and make use of the first-order noise term only. Although this approximation introduces a certain error into our estimator, we find it necessary for the derivation of our estimator, which represents a tight approximation of the maximum likelihood one for small noise power.

Define an auxiliary vector $\zeta=\left[\zeta_{i}\right]^{T}$, such that $\zeta_{i}=$ $\frac{\left(d_{i}-b\right)^{2}-\left\|\boldsymbol{x}-\boldsymbol{a}_{i}\right\|^{2}}{2\left\|\boldsymbol{x}-\boldsymbol{a}_{i}\right\|}$. Then, for sufficiently low measurement noise power, we can disregard the second-order noise term in the above expression (please see $[11,13,22,23]$ and the references therein) and we can approximate $\zeta$ by

$\zeta \sim \mathcal{N}\left(\mathbf{0}_{N \times 1}, \Psi\right)$

where $\boldsymbol{\Psi}=\operatorname{diag}\left\{\left[\sigma_{1}^{2}, \ldots, \sigma_{N}^{2}\right]\right\}$ and $\operatorname{diag}\{\chi\}$ denotes a square diagonal matrix whose main diagonal is composed of elements of $\chi$, and all other elements are zeros. 


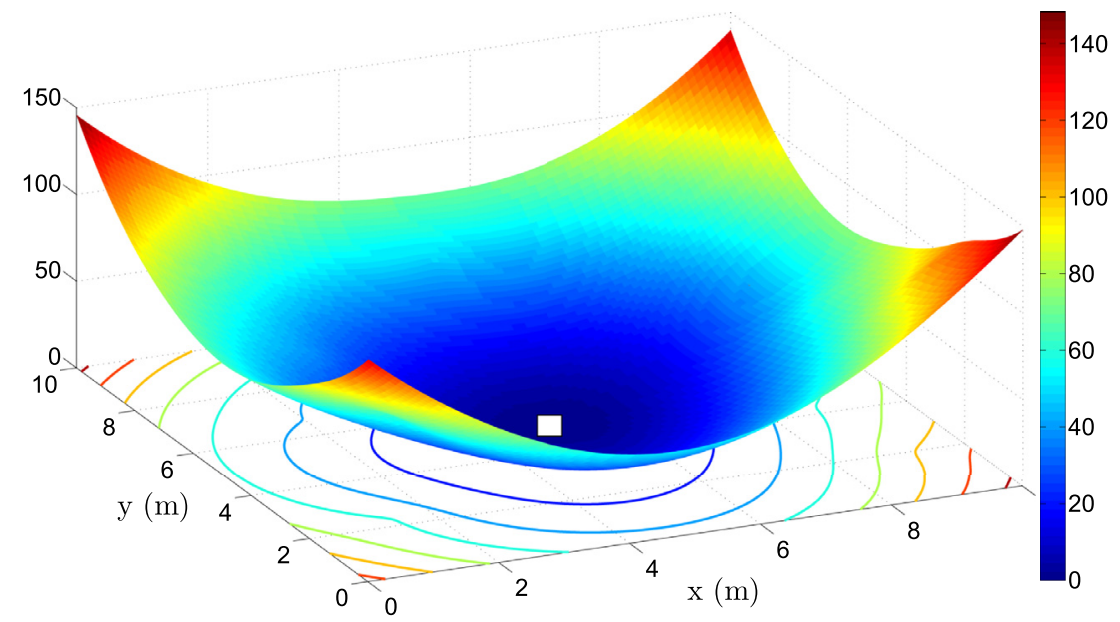

(a) Objective function in (3) using $b_{i}$

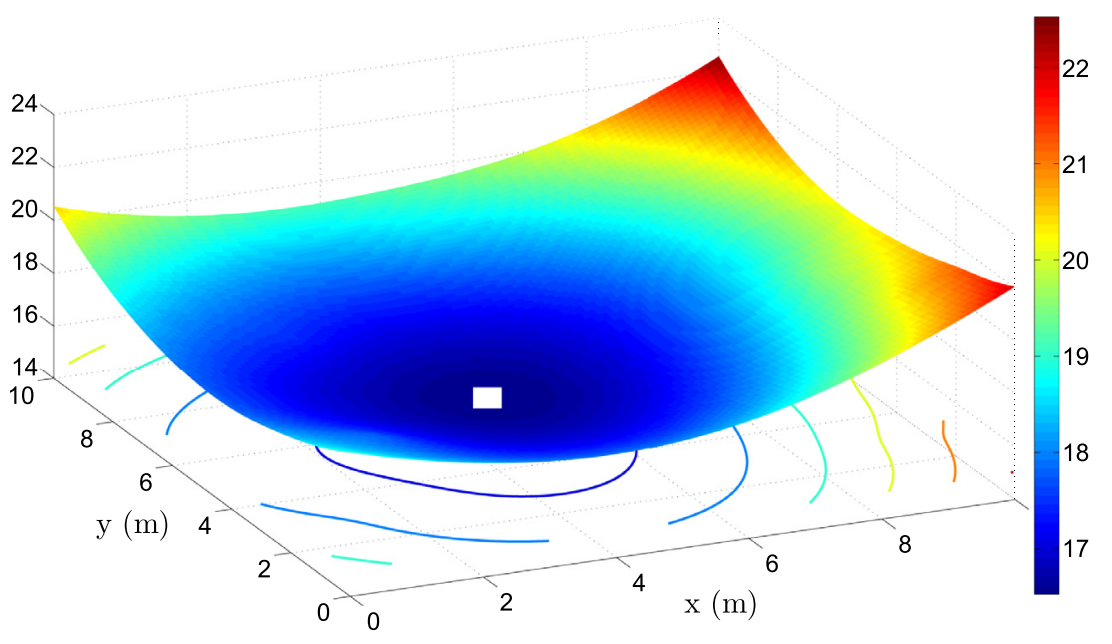

(b) Objective function in (13) using $\widehat{b}$

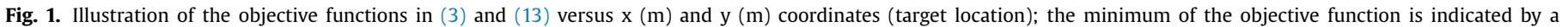
white square.

Then, we can write the PDF of $\zeta$ as

$p(\zeta)=(2 \pi)^{-\frac{N}{2}}|\boldsymbol{\Psi}|^{-\frac{1}{2}} \exp \left\{-\frac{1}{2} \zeta^{T} \boldsymbol{\Psi}^{-1} \zeta\right\}$,

where $|\bullet|$ denotes the determinant of a matrix.

By maximizing the log of the above expression with respect to $\boldsymbol{x}$ and $b$, we get

$(\hat{\boldsymbol{x}}, \hat{b})=\underset{\boldsymbol{x}, b}{\arg \min } \sum_{i=1}^{N}\left(\frac{\left(d_{i}-b\right)^{2}-\left\|\boldsymbol{x}-\boldsymbol{a}_{i}\right\|^{2}}{2\left\|\boldsymbol{x}-\boldsymbol{a}_{i}\right\|}\right)^{2}$.

By close inspection of (8), one can see that both the denominator and nominator are functions of $\boldsymbol{x}$. This problem is highly nonconvex and difficult to solve. Therefore, instead of solving (8), we substitute it by

$(\hat{\boldsymbol{x}}, \hat{b})=\underset{\boldsymbol{x}, b}{\arg \min } \sum_{i=1}^{N}\left(\frac{\left(d_{i}-b\right)^{2}-\left\|\boldsymbol{x}-\boldsymbol{a}_{i}\right\|^{2}}{2 d_{i}}\right)^{2}$.

The intuition behind this is that $d_{i} \geq\left\|\boldsymbol{x}-\boldsymbol{a}_{i}\right\|$, which, translated in words, means that the target's location lies inside the circles defined by the anchors and radii $d_{i}$. Although this approximation is valid for NLOS links in general, it might not be valid for LOS links since the measurement noise may have negative values. Some of the existing works, e.g. [12], tried to minimize the probability of error induced by this approximation by making this constraint somewhat looser by taking advantage of the statistics about the measurement noise. Unlike [12], here, we assume that no knowledge about the variance of the measurement noise is available, and we do not apply any approach of that sort.

If we introduce weights, $w_{i}$, in (9), where $w_{i}=1-d_{i} / \sum_{i=1}^{N} d_{i}$, for $i=1, \ldots, N$, such that more confidence is associated to nearby and/or LOS links, we can rewrite (9) as

$\underset{\boldsymbol{x}, b}{\operatorname{minimize}} \sum_{i=1}^{N} w_{i}\left(\frac{d_{i}^{2}-2 d_{i} b+b^{2}-\left\|\boldsymbol{x}-\boldsymbol{a}_{i}\right\|^{2}}{2 d_{i}}\right)^{2}$,

which can be transformed into a constrained minimization problem as

$\hat{\boldsymbol{y}}=\operatorname{minimize}_{\boldsymbol{y}=\left[\boldsymbol{x}^{T},\|\boldsymbol{x}\|^{2}, b, b^{2}\right]^{T}}\left\{\|\boldsymbol{W}(\boldsymbol{A} \boldsymbol{y}-\boldsymbol{f})\|^{2}: \boldsymbol{y}^{T} \boldsymbol{D} \boldsymbol{y}+2 \mathbf{g}^{T} \boldsymbol{y}=0\right\}$,

where $\boldsymbol{W}=\operatorname{diag}(\widetilde{\boldsymbol{w}})$ and $\boldsymbol{D}=\operatorname{diag}\left(\left[\mathbf{1}_{1 \times k}, 0,1,0\right]\right)$, with $\widetilde{\boldsymbol{w}}=\left[\widetilde{w}_{i}\right]^{T}$ and $\widetilde{w}_{i}=\frac{\sqrt{w_{i}}}{2 d_{i}}$ for $i=1, \ldots, N$, and $\mathbf{1}_{k \times k}$ represents the $k \times k$ matrix 


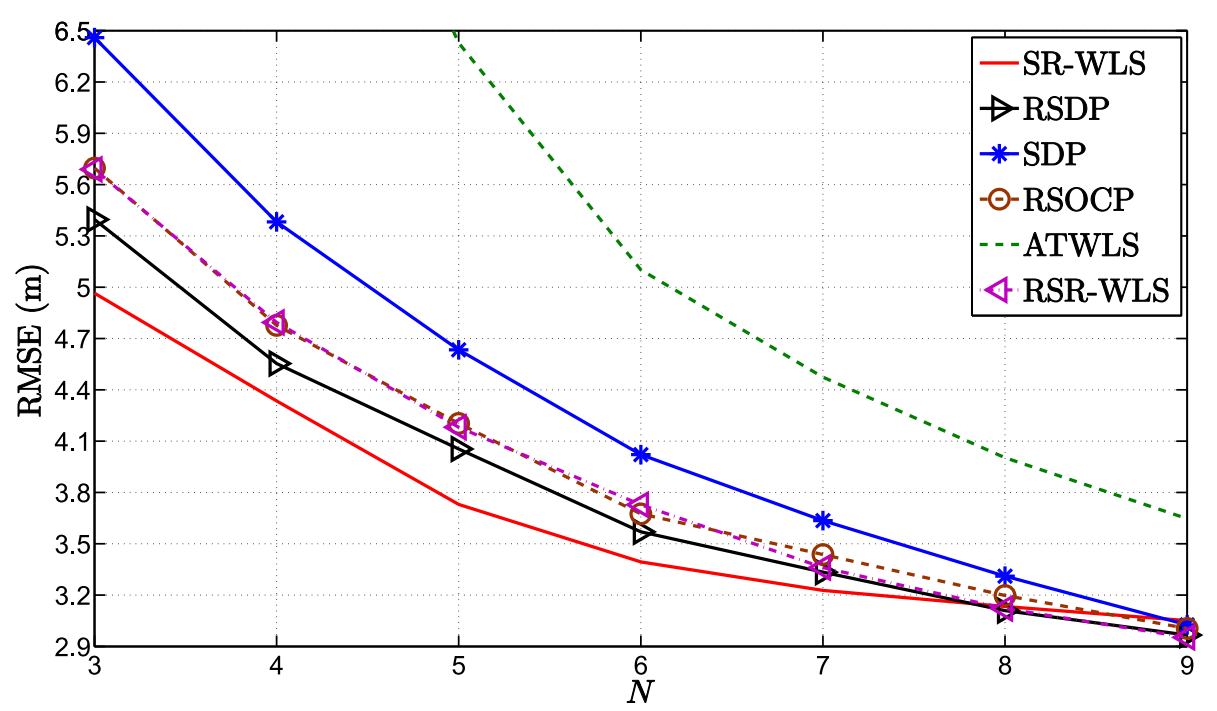

Fig. 2. RMSE versus $N$ comparison, when $b_{i} \sim \operatorname{Exp}\left(\mathcal{U}\left[0, b_{\max }\right]\right) \mathrm{m}, b_{\max }=5 \mathrm{~m}, \sigma_{i}=2 \mathrm{~m},\left|\mathcal{L}_{\mathrm{n}}\right|=N, B=10 \mathrm{~m}, M_{c}=10,000$.

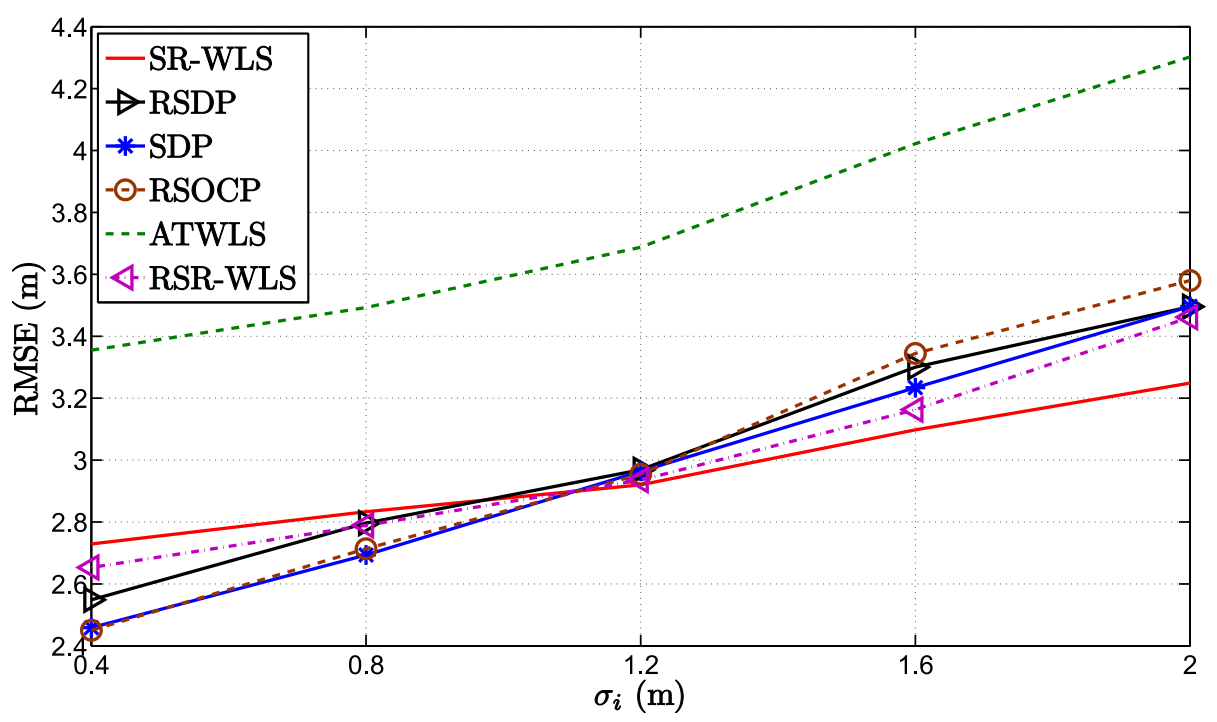

Fig. 3. RMSE versus $\sigma_{i}(\mathrm{~m})$ comparison, when $N=6, b_{i} \sim \operatorname{Exp}\left(\mathcal{U}\left[0, b_{\max }\right]\right) \mathrm{m}, b_{\max }=5 \mathrm{~m},\left|\mathcal{L}_{\mathrm{n}}\right|=4, B=10 \mathrm{~m}, M_{c}=10,000$.

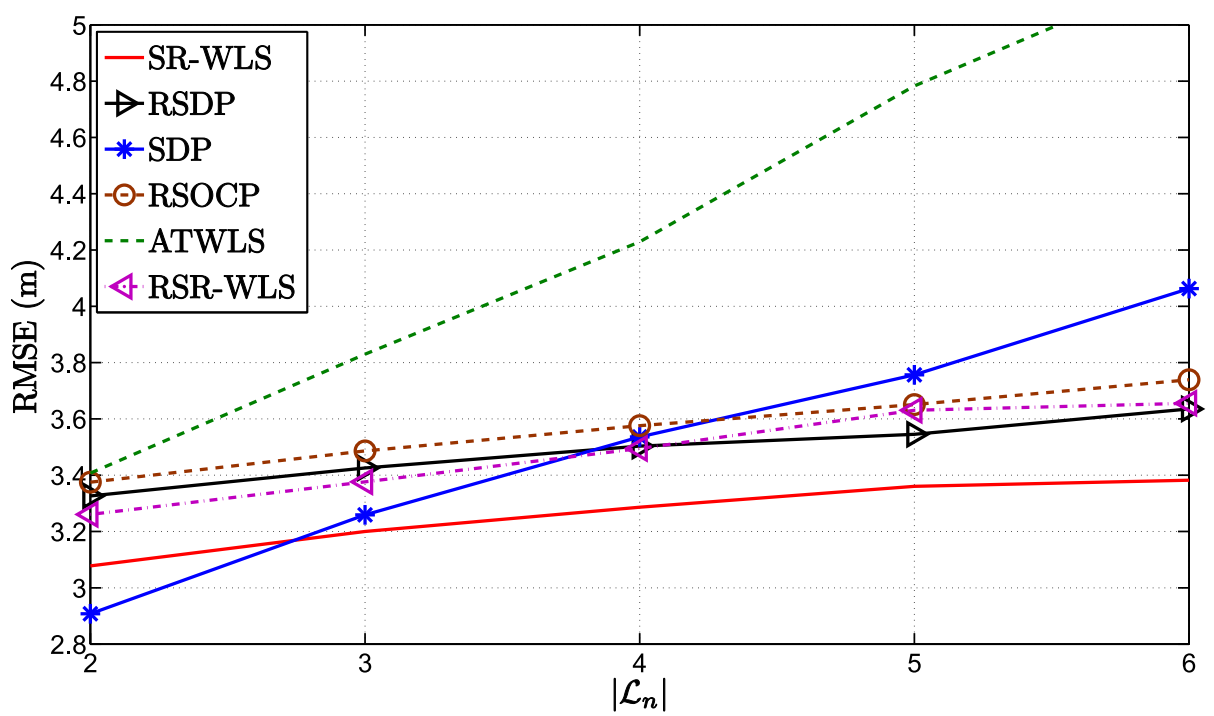

Fig. 4. RMSE versus $\left|\mathcal{L}_{\mathrm{n}}\right|$ comparison, when $N=6, b_{i} \sim \operatorname{Exp}\left(\mathcal{U}\left[0, b_{\max }\right]\right) \mathrm{m}, b_{\max }=5 \mathrm{~m}, \sigma_{i}=2 \mathrm{~m}, B=10 \mathrm{~m}, M_{c}=10,000$. 


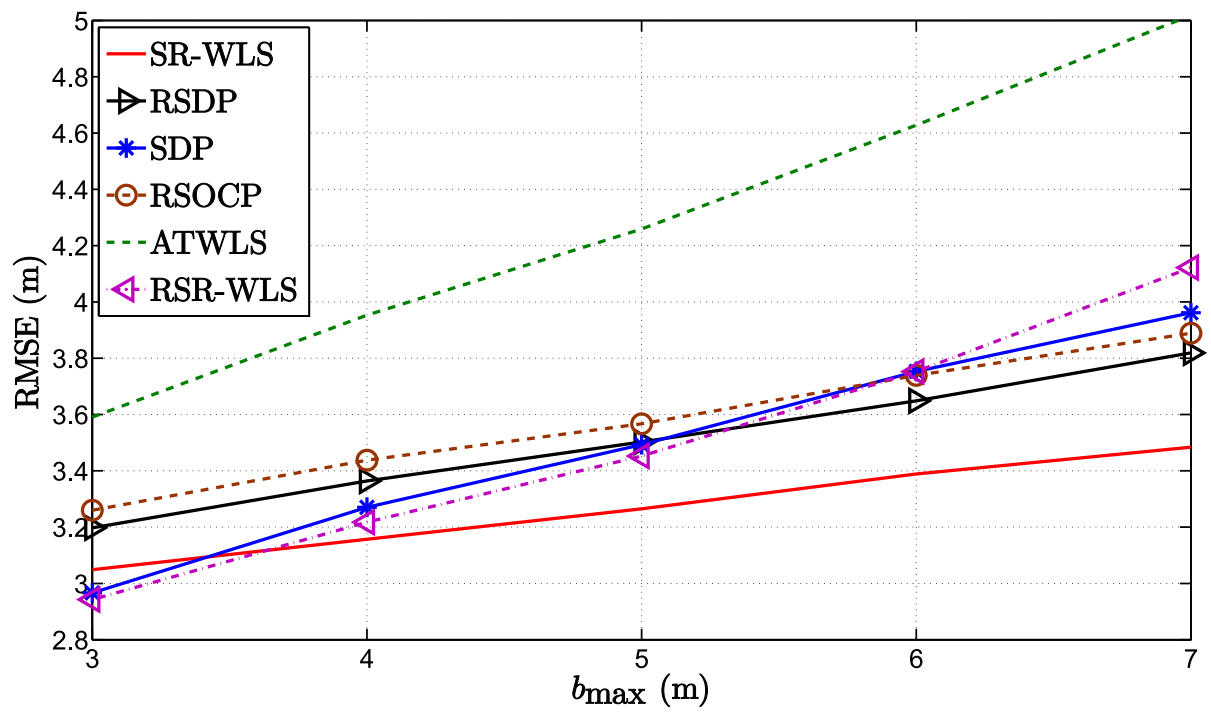

Fig. 5. RMSE versus $b_{\max }(\mathrm{m})$ comparison, when $N=6, b_{i} \sim \operatorname{Exp}\left(\mathcal{U}\left[0, b_{\max }\right]\right) \mathrm{m}, \sigma_{i}=2 \mathrm{~m},\left|\mathcal{L}_{\mathrm{n}}\right|=4, B=10 \mathrm{~m}, M_{c}=10,000$.

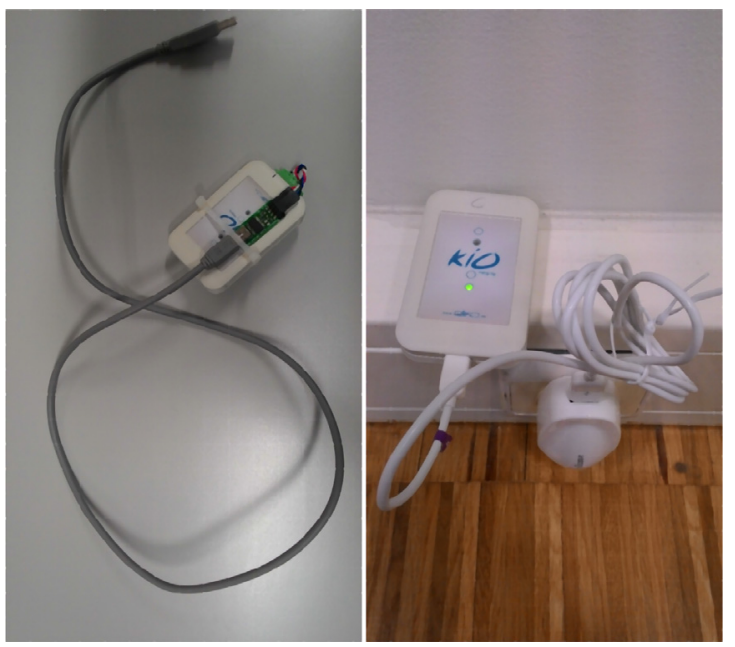

Fig. 6. Equipment used for the experiment: target (left) and anchor (right).

of all ones, and

$\boldsymbol{A}=\left[\begin{array}{llll}2 \boldsymbol{a}_{1}^{T} & -1 & -2 d_{1} & 1 \\ \vdots & \vdots & \vdots & \vdots \\ 2 \boldsymbol{a}_{N}^{T} & -1 & -2 d_{N} & 1\end{array}\right], \boldsymbol{f}=\left[\begin{array}{l}\left\|\boldsymbol{a}_{1}\right\|^{2}-d_{1}^{2} \\ \vdots \\ \left\|\boldsymbol{a}_{N}\right\|^{2}-d_{N}^{2}\end{array}\right], \boldsymbol{g}=\left[\begin{array}{l}\mathbf{0}_{k \times 1} \\ -1 / 2 \\ 0 \\ -1 / 2\end{array}\right]$

Notice that the constraint in (11) essentially says that: $\boldsymbol{x}^{T} \boldsymbol{x}=$ $\|\boldsymbol{x}\|^{2}$ and $b b=b^{2}$, only rewritten in a vector form. It is written deliberately in a quadratic form, such that, together with the quadratic objective function, it falls into a category of problems known in the literature as GTRS [20]. This type of problems, even though non-convex in general, has a convenient characteristic of being strictly decreasing over an interval that can be readily calculated. Hence, the exact solution to the problem in (11) can be easily acquired by just a bisection procedure [20].

The solution obtained from (11) can then be exploited to find an ML estimate of $b, \widehat{b}$, as

$\widehat{b}=\frac{\sum_{i=1}^{N}\left(d_{i}-\left\|\hat{\boldsymbol{x}}-\boldsymbol{a}_{i}\right\|\right)}{N}$.

Then, we can take advantage of this estimate to further enhance the accuracy of the target location estimation, i.e., from (10) we can write

$\underset{\boldsymbol{x}}{\operatorname{minimize}} \sum_{i=1}^{N} w_{i}\left(\frac{d_{i}^{2}-2 d_{i} \widehat{b}+\widehat{b}^{2}-\left\|\boldsymbol{x}-\boldsymbol{a}_{i}\right\|^{2}}{2 d_{i}}\right)^{2}$,

which can be rewritten in a vector form similar as in (11), i.e.,

$\hat{\boldsymbol{y}}=\operatorname{minimize}_{\widetilde{\boldsymbol{y}}=\left[\boldsymbol{x}^{T},\|\boldsymbol{x}\|^{2}\right]^{T}}\left\{\|\boldsymbol{W}(\tilde{\boldsymbol{A}} \widetilde{\boldsymbol{y}}-\tilde{\boldsymbol{f}})\|^{2}: \widetilde{\boldsymbol{y}}^{T} \widetilde{\boldsymbol{D}} \widetilde{\boldsymbol{y}}+2 \widetilde{\boldsymbol{g}}^{T} \widetilde{\boldsymbol{y}}=0\right\}$,

where

$\widetilde{\boldsymbol{A}}=\left[\begin{array}{ll}2 \boldsymbol{a}_{1}^{T} & -1 \\ \vdots & \vdots \\ 2 \boldsymbol{a}_{N}^{T} & -1\end{array}\right], \widetilde{\boldsymbol{f}}=\left[\begin{array}{l}\left\|\boldsymbol{a}_{1}\right\|^{2}-d_{1}^{2}+2 d_{1} \widehat{b}-\widehat{b}^{2} \\ \vdots \\ \left\|\boldsymbol{a}_{N}\right\|^{2}-d_{N}^{2}+2 d_{N} \widehat{b}-\widehat{b}^{2}\end{array}\right]$,

$\widetilde{\boldsymbol{D}}=\left[\begin{array}{ll}\mathbf{I}_{k} & \mathbf{0}_{k \times 1} \\ \mathbf{0}_{1 \times k} & 0\end{array}\right], \widetilde{\mathbf{g}}=\left[\begin{array}{l}\mathbf{0}_{k \times 1} \\ -1 / 2\end{array}\right]$.

In order to check the validity of the applied approximations and the tightness of the derived estimator in (13) in comparison with the ML one in (3), we illustrate a realization of the respective objective functions in Fig. 1. In the figure, the true location of the target was set at $[5,5]^{T}$, and $N=10$ anchors were randomly deployed in a $10 \times 10 \mathrm{~m}^{2}$ region, with $\sigma_{i}=0 \mathrm{~m}$ and the rest of the parameters set as in Section 5. The global minimum of the objective functions is denoted by a white square. It can be seen from Fig. 1, that the global minimum of the objective function in (13), $[4.8,5.1]^{T}$, corresponds closely to minimum of the objective function in (3), $[4.9,4.9]^{T}$. Furthermore, it is worth mentioning that, in contrast to the existing convex optimization-based techniques [11-14], here we do not apply any further relaxations to our derived non-convex estimator, but solve it exactly.

Explicit summary of the proposed bisection-based procedure, in the remaining text called "SR-WLS", is given in Algorithm 1. Note that at lines $4-6$ we use the general assumption that the NLOS bias is positive $[11-14,18,19]$, i.e., if $\widehat{b}<0$, we treat all the links as LOS $(\widehat{b}=0)$.

\section{Complexity analysis}

Assuming that $K$ is the maximum number of steps in the proposed bisection-based procedure, Table 1 provides an overview of the considered algorithms together with their worst case computational complexities. 


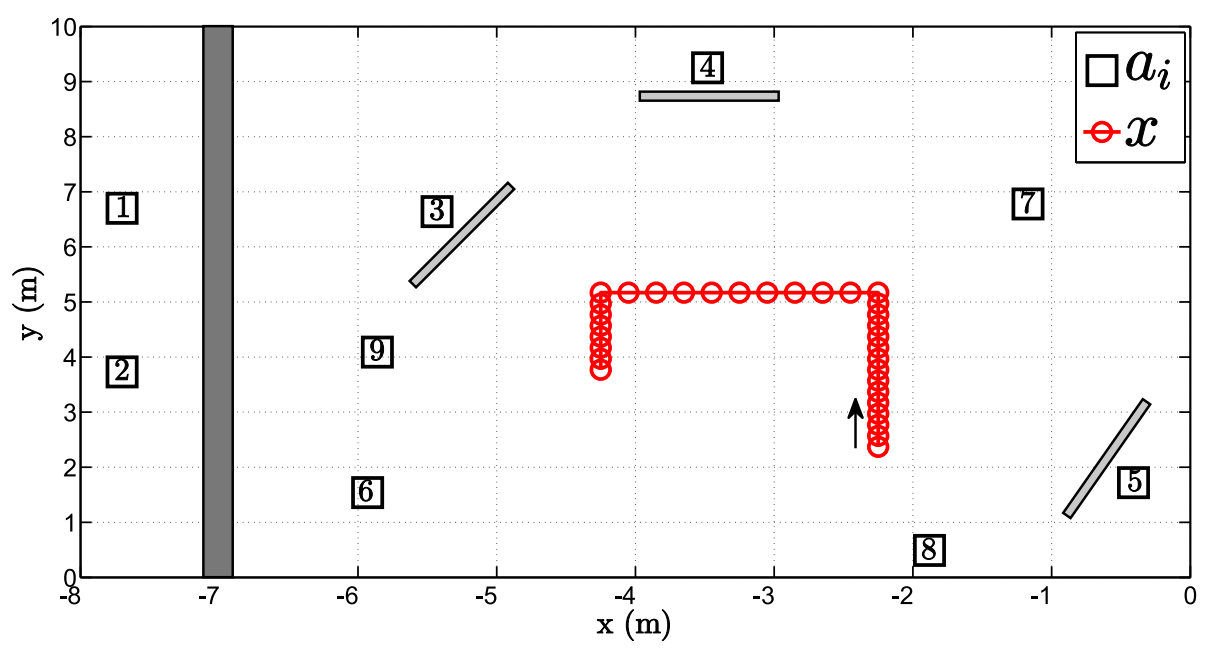

Fig. 7. Experimental set-up with 9 anchors (squares) and 32 targets (circles).

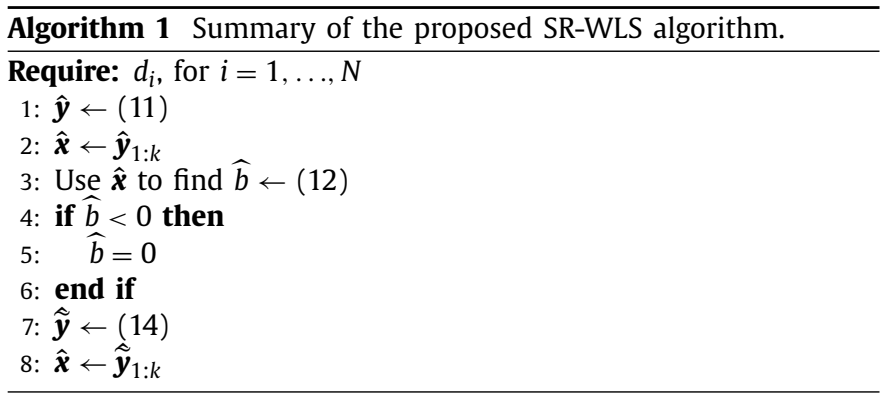

Table 1

Summary of the considered algorithms.

\begin{tabular}{lll}
\hline Algorithm & Description & Complexity \\
\hline SR-WLS & The proposed bisection method & $2 \times \mathcal{O}(K N)$ \\
RSDP & The robust SDP method in [11] & $\mathcal{O}\left(N^{6.5}\right)$ \\
SDP & The SDP method in [12] & $\mathcal{O}\left(N^{3}\right)$ \\
RSOCP & The robust SOCP method in [13] & $\mathcal{O}\left(N^{3.5}\right)$ \\
ATWLS & The adaptive WLS method in [18] & $\mathcal{O}(N)$ \\
RSR-WLS & The robust GTRS method in [19] & $\mathcal{O}(K N)$ \\
\hline
\end{tabular}

From Table 1, one can see that the computational complexity of all methods depends mainly on the network size, i.e., the number of anchors. Table 1 shows that the computational complexities of the SR-WLS, ATWLS and RSR-WLS methods are linear in N, while the complexity of other existing methods is significantly higher.

\section{Performance results}

\subsection{Simulation results}

This section verifies the performance of the proposed algorithm through computer simulations. Its performance is compared with the existing ones, ${ }^{1}$ summarized in Table 1 . The radio measurements were generated by using (1). All sensors were deployed randomly inside a quadratic region with a side length $B=20 \mathrm{~m}$ in each Monte Carlo, $M_{c}$, run. The NLOS bias was drawn from an exponential distribution whose mean was drawn from a uniform

\footnotetext{
${ }^{1}$ Note that here we do not include the PESDP algorithm described in [14] for comparison. The reason is that it was designed for cooperative localization where the perturbed SDP relaxation was derived for target/target connections only. Since here we take into account target/anchor connections exclusively, these perturbation terms are useless, which affects the performance of the PESDP.
}

distribution on an interval $\left[0, b_{\max }\right]$, i.e., $b_{i} \sim \operatorname{Exp}\left(\mathcal{U}\left[0, b_{\max }\right]\right)$ for $i=1, \ldots, N$. Furthermore, for the proposed method, $K=30$ is used. The main performance metric used here is the root mean square error (RMSE), defined as RMSE $=\sqrt{\sum_{i=1}^{M_{c}} \frac{\left\|\boldsymbol{x}_{i}-\widehat{\boldsymbol{x}}_{i}\right\|^{2}}{M_{c}}}$, where $\widehat{\boldsymbol{x}}_{i}$ denotes the estimate of the true target location, $\boldsymbol{x}_{i}$, in the $i$ th $M_{c}$ run.

Fig. 2 illustrates the RMSE versus $N$ performance comparison of the considered algorithms, for $\sigma_{i}=2 \mathrm{~m}$ and $\left|\mathcal{L}_{\mathrm{n}}\right|=4$, where $|\bullet|$ denotes the cardinality of a set. The figure exhibits improvement of all algorithm as $N$ is increased. This behavior is intuitive, since more reliable information is added into the network with every anchor and all algorithms profit from it. Nevertheless, the network cost is directly proportional to $N$, and in practice we want to keep $N$ as low as possible. Even though the proposed algorithm outperforms the state-of-the-art methods for almost every $N$, it is exactly for low $N$ that the new algorithm achieves the most significant error reduction. Fig. 3 illustrates the RMSE versus $\sigma_{i}(\mathrm{~m})$ performance comparison of the considered algorithms, for $N=6$ and $\left|\mathcal{L}_{\mathrm{n}}\right|=4$. This figure shows that the performance of all algorithms deteriorates as $\sigma_{i}$ grows, as anticipated. It also reveals that the proposed algorithm matches the performance of the more computationally complex ones, and even outperforms them for medium-to-high $\sigma_{i}$. Moreover, although the ATWLS algorithm assumes perfect knowledge about $\sigma_{i}$ and which links are LOS/NLOS, the new algorithm performs significantly better than this linear method throughout all range of $\sigma_{i}$. Fig. 4 illustrates the RMSE versus $\left|\mathcal{L}_{\mathrm{n}}\right|$ performance comparison of the considered algorithms, for fixed $N=6$ and $\sigma=2 \mathrm{~m}$. The figure exhibits excellent mitigation capacity of the NLOS bias of the new algorithm, since it suffers a relatively small performance deterioration $(\approx 20 \%$ ) when all links are NLOS in comparison with the starting setting; this result is particularly important, since our algorithm is not a robust one, but its mitigation capability is competitive with those from $[11,13,19]$. Furthermore, one can see from Fig. 4 that the SDP performs best when $\left|\mathcal{L}_{\mathrm{n}}\right|$ is low, but its performance is highly affected with the increase of $\left|\mathcal{L}_{\mathrm{n}}\right|$. Fig. 5 illustrates the RMSE versus $b_{\max }(\mathrm{m})$ performance comparison of the considered algorithms, for fixed $N=6$ and $\sigma=2 \mathrm{~m}$ and $\left|\mathcal{L}_{\mathrm{n}}\right|=4$. As expected, the performance of all algorithms impairs as $b_{\max }$ grows. From Fig. 5 , we can see that the proposed algorithm matches the performance of the much more complex ones, while in general it outperforms considerably the existing linear ones. Finally, it is also noteworthy that, in our simulations, the proposed algorithm provided always a valid solution to the problem, while most of the existing algorithms had suffered some infeasible runs. To illustrate this, in Table 2, we present the 


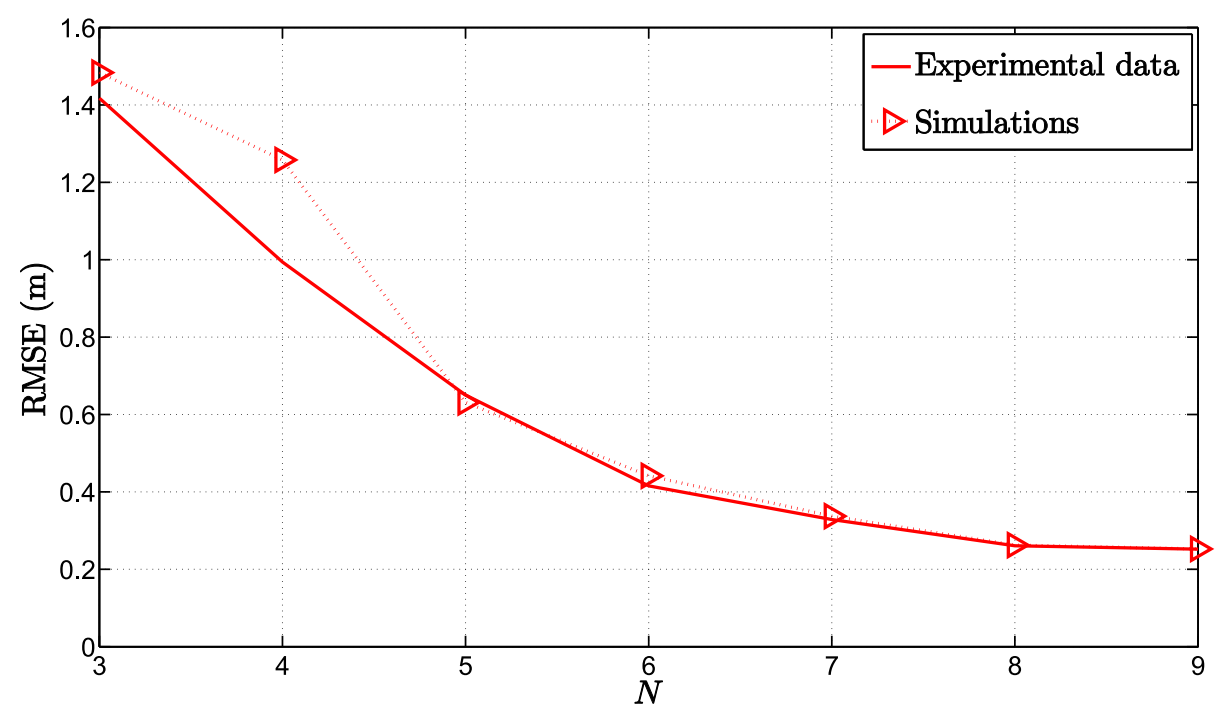

Fig. 8. RMSE versus $N$ comparison.

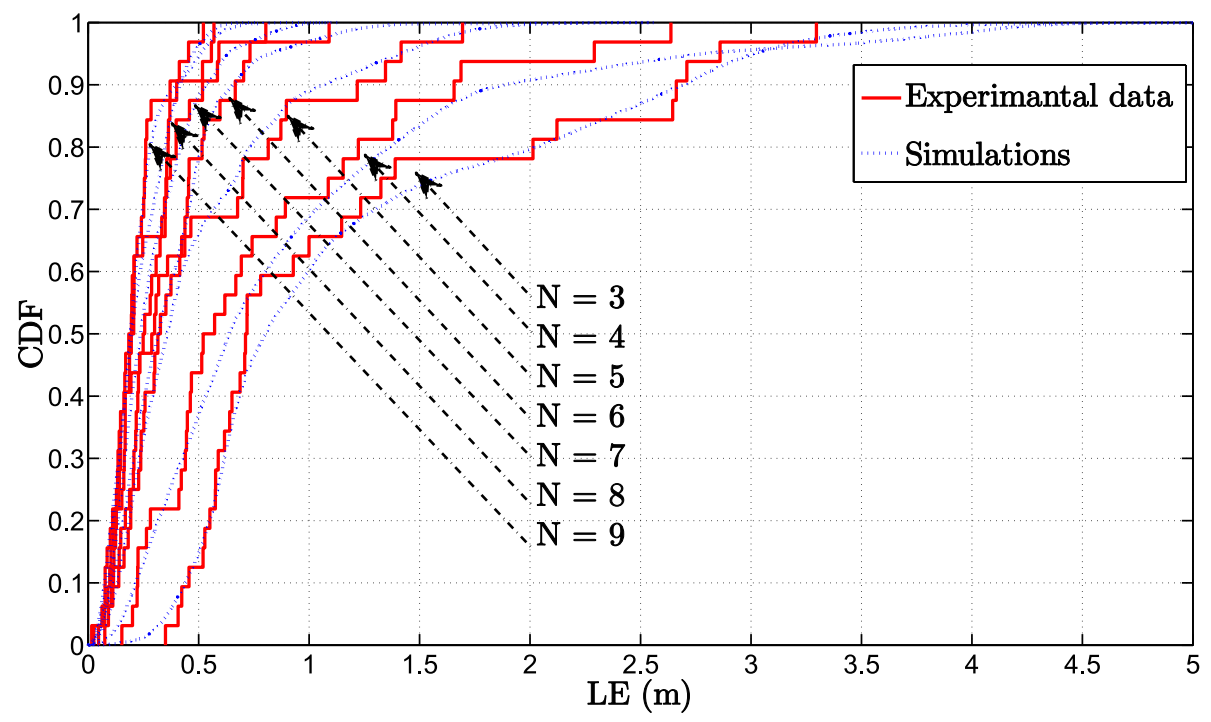

Fig. 9. CDF versus LE (m) comparison for different $N$.

Table 2

The average running time and the number of infeasible runs (NIR) for $N=$ 6, $\left|\mathcal{L}_{n}\right|=4, \sigma=2 \mathrm{~m}, M_{c}=10,000$. CPU: Intel(R)Core(TM)i7-4710HQ $2.0 \mathrm{GHz}$ and 16GB RAM.

\begin{tabular}{lllllll}
\hline Algorithm & SR-WLS & RSDP & SDP & RSOCP & ATWLS & RSR-WLS \\
\hline Time (s) & 0.0026 & 0.43 & 0.37 & 0.42 & 0.0007 & 0.0014 \\
NIR & 0 & 6082 & 231 & 6105 & 0 & 0 \\
\hline
\end{tabular}

average execution time per $M_{c}$ run of the considered algorithms, ${ }^{2}$ as well as the number of infeasible runs (NIR) for each of them. ${ }^{3}$ From it, it can be seen that the proposed algorithm is the third fastest, which is in concordance with the results in Table 1, and that its solution is always feasible, while the same can not be said for the more complex existing algorithms.

\footnotetext{
2 The existing methods were implemented using MATLAB package CVX [24], were the solver was set as it was stated in each specific work.

${ }^{3}$ Note that the results presented in Figs. 2-5 are all for $M_{c}=10,000$ feasible cases.
}

\subsection{Experimental results}

In this section, we validate the performance of the proposed algorithm through the use of real-indoor experimental data. The data was collected by using KIO Real Time Location System equipment [25], which follows the IEEE802.15.4-2011-UWB communication standard and extracts the distance information from the time of flight measurements. In Fig. 6, we present the devices used: the target (left) and an anchor (right). The KIO kit included three anchors and a tag (target), which we used repeatedly at various locations in order to produce a realistic indoor scenario, as shown in Fig. 7. The measurement campaign was realized inside the Instituto Superior Técnico building at Taguspark campus, Oeiras, Portugal.

Fig. 7 illustrates the considered experimental set-up, comprising $N=9$ anchors and 32 different locations of the target, respectively denoted by black squares and red circles. The true locations of the anchors are given in Table 3, while the true initial target location was set at $[-2.25,2.37]^{T} \mathrm{~m}$. The target was then moved by taking a step of $0.2 \mathrm{~m}$ length in a direction indicated by a black arrow in Fig. 7. The LOS of the first five anchors was blocked by either a building wall or a metal cabinet represented by a dark grey rectan- 
Table 3

True locations of anchors in the experimental set-up.

\begin{tabular}{llllllllll}
\hline$i$ & 1 & 2 & 3 & 4 & 5 & 6 & 7 & 8 & 9 \\
\hline $\boldsymbol{a}_{i}(\mathrm{~m})$ & -7.7 & -7.7 & -5.43 & -3.48 & -0.41 & -5.93 & -1.17 & -1.88 & -5.86 \\
& 6.7 & 3.73 & 6.64 & 9.25 & 1.72 & 1.54 & 6.78 & 0.49 & 4.09 \\
\hline
\end{tabular}

gle and grey rectangles in Fig. 7, respectively, while the remaining four anchors had unimpeded LOS to the target.

Fig. 8 illustrates the RMSE versus $N$ performance comparison of the proposed algorithm tested against the real-indoor experimental data and simulations in the considered experimental setting. Note that we always used the first $N$ anchors from Table 3 for any $N$ in Fig. 8. The results shown in this figure corroborate that the proposed algorithm works excellent for all $N$, offering good estimation accuracy in a real-indoor scenario. Furthermore, it can be seen from the figure that the simulation results correspond closely to the results obtained by using experimental data.

Fig. 9 illustrates the cumulative distribution function (CDF) of localization error (LE) versus $N$ of the proposed algorithm in the considered experimental setting. Again, the first $N$ anchors from Table 3 were used for any $N$ in Fig. 9. The LE is defined as LE = $\left\|\boldsymbol{x}_{i}-\widehat{\boldsymbol{x}}_{i}\right\|$, for $i=1, \ldots, M_{c}$. From the figure, it can be seen that the median LE for $N=9$ is $\mathrm{LE}<0.2 \mathrm{~m}$, and that the proposed algorithm achieves $L E \leq 1 \mathrm{~m}$ in almost $90 \%$ of the cases when all $N=5$ links are NLOS.

\section{Conclusions}

In this work, we have presented a novel range-based algorithm for target localization in harsh indoor environments. The new method does not require a priori knowledge about LOS and NLOS links, neither the knowledge of the statistics about the NLOS bias (not even the magnitude of the NLOS bias, as some existing algorithms do). By employing a SR approach and WLS criterion, we have mitigated the negative effect of the NLOS bias, and we have developed an efficient estimator whose exact solution is obtained merely by a bisection procedure. A great advantage of the proposed algorithm over the majority of the existing ones is its linear computational complexity in $N$. The simulation results have confirmed that the mitigation capability of the NLOS bias of our algorithm is excellent, and that its performance not only matches the performance of the existing ones, but slightly surpasses it in general. Finally, the proposed algorithm was also validated by using a real-indoor experimental data. The results confirmed excellent estimation accuracy of the new approach.

\section{Acknowledgment}

This work was partially supported by Fundação para a Ciência e a Tecnologia under Project PEst-OE/EEI/UI0066/2014 (UNINOVA), Project UID/EEA/ 50009/2013, and Program Investigador FCT under Grant IF/00325/2015.

The authors would also like to express their gratitude to Vasco Rato, as well as Prof. Dr. João Pedro Gomes and Prof. Dr. João Paulo
Costeira from IST-UL for allowing us to use the equipment and for providing us a great help within the actual measurement process.

\section{References}

[1] G. Ismail, C.C. Chong, A survey on TOA based wireless localization and NLOS mitigation techniques, IEEE Commun. Surv. Tuts. 11 (3) (2009) 107-124.

[2] S. Tomic, M. Beko, R. Dinis, Distributed RSS-based localization in wireless sensor networks based on second-order cone programming, Sensors 14 (10) (2014) 18410-18432.

[3] S. Zhang, M.J. Er, B. Zhang, Y. Naderahmadian, A novel heuristic algorithm for node localization in anisotropic wireless sensor networks with holes, Signal Process. 138 (2017) 27-34.

[4] S. Tomic, M. Beko, R. Dinis, RSS-based localization in wireless sensor networks using convex relaxation: Noncooperative and cooperative schemes, IEEE Trans. Veh. Technol., 64 (5) (2015) 2037-2050.

[5] S. Tomic, M. Beko, R. Dinis, 3-d target localization in wireless sensor network using RSS and aoa measurement, IEEE Trans. Veh. Technol. 66 (4) (2017) $3197-3210$

[6] X. Qua, L. Xie, An efficient convex constrained weighted least squares source localization algorithm based on TDOA measurements, Signal Process. 119 (2016) 142-152.

[7] S. Tomic, M. Beko, R. Dinis, P. Montezuma, Distributed algorithm for target localization in wireless sensor networks using RSS and aoa measurements, Pervasive Mobile Comput. 37 (2016) 63-77.

[8] S. Tomic, M. Beko, R. Dinis, Distributed RSS-aoa based localization with unknown transmit powers, IEEE Wirel. Commun. Lett. 5 (4) (2016) 392-395.

[9] B.K. Chalisea, Y.D. Zhanga, M.G. Amina, B. Himed, Target localization in a multi-static passive radar system through convex optimization, Signal Process. 102 (2014) 207-215.

[10] S. Tomic, M. Beko, R. Dinis, P. Montezuma, A closed-form solution for RSS/aoa target localization by spherical coordinates conversion, IEEE Wirel. Commun. Lett. 5 (6) (2016) 680-683.

[11] G. Wang, H. Chen, Y. Li, N. Ansari, NLOS error mitigation for TOA-based localization via convex relaxation, IEEE Trans. Wirel. Commun. 13 (8) (2014) 4119-4131.

[12] R.M. Vaghefi, R.M. Buehrer, Cooperative localization in NLOS environments using semidefinite programming, IEEE Commun. Lett. 19 (8) (2015) 1382-1385.

[13] S. Zhang, S. Gao, G. Wang, Y. Li, Robust NLOS error mitigation method for TOA-based localization via second-order cone relaxation, IEEE Commun. Lett. 19 (12) (2015) 2210-2213.

[14] P.M. Ghari, R. Shahbazian, S.A. Ghorashi, Wireless sensor network localization in harsh environments using SDP relaxation, IEEE Commun. Lett. 20 (1) (2016) 137-140.

[15] K. Yu, Y.G. Guo, Improved positioning algorithms for nonline-of-sight environments, IEEE Trans. Veh. Technol. 57 (4) (2008) 2342-2353.

[16] I. Guvenc, C.C. Chong, F. Watanabe, H. Inamura, NLOS identification and weighted least-squares localization for UWB systems using multipath channel statistics, EURASIP J. Adv. Signal Process. 2008 (2007) 1-14.

[17] S. Marano, W.M. Gifford, H. Wymeersch, M.Z. Win, NLOS identification and mitigation for localization based on UWB experimental data, IEEE J. Sel. Areas Commun. 28 (7) (2009) 1026-1035.

[18] C.H. Park, J.H. Chang, Robust time-of-arrival source localization employing error covariance of sample mean and sample median in line-of-sight/non-line-of-sight mixture environments, EURASIP J. Adv. Signal Process. 2016 (89) (2016) 1-11.

[19] S. Tomic, M. Beko, R. Dinis, P. Montezuma, A robust bisection-based estimator for TOA-based target localization in NLOS environments, 2017, To appear in IEEE Commun. Letters.

[20] A. Beck, P. Stoica, J. Li, Exact and approximate solutions of source localization problems, IEEE Trans. Sig. Process., 56 (5) (2008) 1770-1778.

[21] S.M. Kay, Fundamentals of Statistical Signal Processing: Estimation Theory., Prentice-Hall: Upper Saddle River, NJ, USA, 1993.

[22] Y.T. Chan, K.C. Ho, A simple and efficient estimator for hyperbolic location, IEEE Trans. Signal Process. 42 (8) (1994) 1905-1915.

[23] K.W. Cheung, H.C. So, W.K. Ma, Y.T. Chan, M. Jin, Least squares algorithms for time-of-arrival-based mobile location, IEEE Trans. Signal Process. 52 (4) (2004) 1121-1128.

[24] M. Grant, S. Boyd, CVX: Matlab Software for Disciplined Convex Programming Version 1.21. Available online: http://cvxr.com/cvx (accessed on 15 April 2010).

[25] Precise UWB-based real time location system, OU Eliko Tehnoloogia Arenduskeskus, Tallin, Estonia: http://www.eliko.ee/products/kio-rtls/. 